\title{
CoRoT target HD 51844: a $\delta$ Scuti star in a binary system with periastron brightening $\dagger$
}

\section{Markus Hareter and Margit Paparó}

\author{
Konkoly Observatory MTA CSFK \\ Konkoly Thege M. út 15-17, H-1121 Budapest, Hungary \\ email: hareter@konkoly.hu, paparo@konkoly.hu
}

\begin{abstract}
The star HD 51844 was observed in the CoRoT LRa02 as a target in the seismology field, which turned out to be an SB2 system. The 117-day light curve revealed $\delta$ Scuti pulsations in the range of 6 to $15 \mathrm{~d}^{-1}$ where four frequencies have amplitudes larger than $1.4 \mathrm{mmag}$, and a rich frequency spectrum with amplitudes lower than 0.6 mmag. Additionally, the light curve exhibits a 3 -mmag brightening event recurring every 33.5 days with a duration of about 5 days. The radial velocities from spectroscopy confirmed that the star is an eccentric binary system with nearly identical masses and physical parameters. The brightening event in the light curve coincides with the maximum radial-velocity separation showing that the brightening is in fact caused by tidal distortion and/or reflected light. One component displays large line-profile variations, while the other does not show significant variation. The frequency analysis revealed a quintuplet structure of the four highest-amplitude frequencies, which is due to the orbital motion of the pulsating star.
\end{abstract}

Keywords. stars: individual: HD 51844, stars: variables: $\delta$ Scuti, binaries: spectroscopic

\section{Introduction}

Pulsating components of binary systems are of special interest because the masses of the components can be derived directly if the inclination angle $i$ can be constrained sufficiently well. These masses can then be compared to theoretical predictions from evolution theory and pulsation theory.

Thompson et al. (2012) propose a class of eccentric binary systems with dynamic tidal distortions found among Kepler stars. Typical for this class is a periastron brightening of various forms akin to an electrocardiogram. Hence, they coined the term "heartbeat stars" for this class. Such stars may or may not show eclipses. The light variation allows to constrain orbital elements such as orbital period, inclination, argument of periastron and eccentricity.

$\dagger$ Based on observations made with the Mercator Telescope, operated on the island of La Palma by the Flemish Community, at the Spanish Observatorio del Roque de los Muchachos of the Instituto de Astrofísica de Canarias. Based on observations obtained with the HERMES spectrograph, which is supported by the Fund for Scientific Research of Flanders (FWO), Belgium, the Research Council of K.U. Leuven, Belgium, the Fonds National Recherches Scientific (FNRS), Belgium, the Royal Observatory of Belgium, the Observatoire de Genève, Switzerland and the Thüringer Landessternwarte Tautenburg, Germany. Based on CoRoT space-based photometric data; the CoRoT space mission was developed and operated by the French space agency CNES, with the participation of ESA's RSSD and Science Programmes, Austria, Belgium, Brazil, Germany, and Spain. Based on observations collected at La Silla Observatory, ESO (Chile) with the HARPS spectrograph at the 3.6-m telescope, under programme LP185.D-0056. 


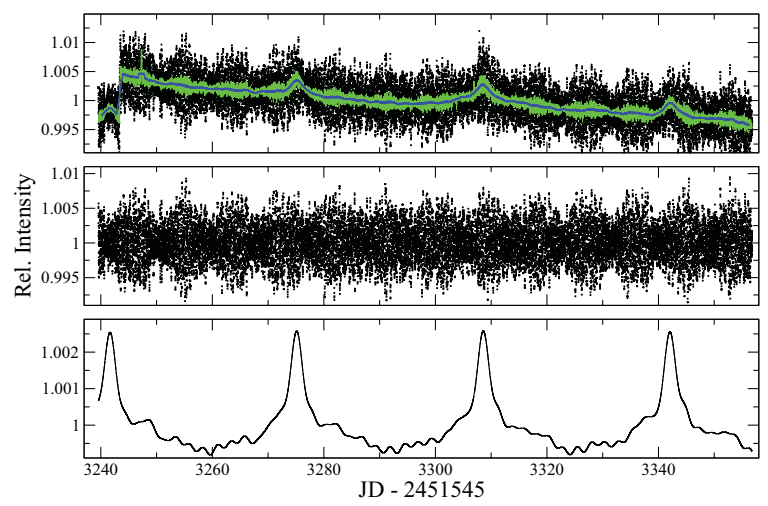

Figure 1. CoRoT N2 light curve (top panel, full dots) together with running averages (300 and 3000 points) to highlight the pulsation and the light variation due to the binarity and instrumental effects. To obtain the pure pulsation light curve (middle panel), the binary model (shown in the bottom panel) was subtracted. Note that the y-axes are of different scales.

\section{The CoRoT light curve}

The binary HD 51844 was observed in the CoRoT LRa02 for 117 days continuously. The high-quality light curve shows multi-mode $\delta$ Sct pulsation and a periodic brightening event. Figure 1 shows the CoRoT light curve together with two running averages (300 and 3000 points) to illustrate the p-mode pulsation and the light variation due to the orbital motion of the stars. The outliers and jumps were removed in an iterative approach by involving prewhitening of the $\delta$ Sct-type pulsations and correcting the residuals. The removal of outlying data points involved a 3- $\sigma$ clipping on the residuals rather than the original data. Since the pulsation frequencies are well separated from the binary light variation, we constructed a simple binary light curve by calculating a fit using the first fifteen harmonics of the orbital frequency.

\section{Pulsation and frequency modulation}

The subsequent frequency analysis was performed on the corrected data where the binary model was subtracted. In this paper, we focus on the effects of the binarity. A more detailed frequency analysis will be published elsewhere, though a brief summary is given below.

The frequency analysis using SigSpec (Reegen 2007) resulted in more than 700 peaks using a significance limit of 6 , roughly corresponding to a signal-to-noise ratio $(\mathrm{S} / \mathrm{N})$ of 4 in the case of white noise. The pulsation frequencies range from 5 to $15 \mathrm{~d}^{-1}$ where four frequencies have amplitudes larger than $1.4 \mathrm{mmag}$ (in the integrated light from both components). These dominant frequencies $\left(f_{1}\right.$ to $\left.f_{4}\right)$ are equal to $12.213,7.054$, 6.943 and $8.141 \mathrm{~d}^{-1}$, respectively. There are closely spaced frequencies around the two dominant modes $\left(f_{1}\right.$ and $\left.f_{2}\right)$ forming distinctive groups. $f_{4}$ is a single frequency outside of the groups and reveals an exact $3 / 2$ ratio to the dominant mode. This ratio was also detected among a few frequencies with lower amplitude.

The high-quality of the CoRoT data in the seismology field allows one to detect frequency modulation due to the orbital motion. In an eccentric binary system a more complex structure of the side-lobes is expected (Shibahashi \& Kurtz 2012). In our case we find side-peaks around the four dominant frequencies spaced by the orbital frequency and sometimes twice the orbital frequency, hereafter referred to as the first- and second-order side-lobes. The amplitudes of the latter side-lobes range from 6.7 to $20 \mathrm{ppm}$. The average 
Table 1. Frequencies, amplitudes and phases used for the determination of theoretical RV curves.

\begin{tabular}{|c|c|c|c|c|c|c|c|c|c|c|c|c|c|c|}
\hline$f_{c}$ & $f_{1+}$ & {$\left[\mathrm{d}^{-1}\right]$} & $f_{2+}$ & $f_{2-}$ & $a_{c}$ & $a_{1+}$ & $\begin{array}{c}a_{1-} \\
{[\mathrm{ppm}]}\end{array}$ & $a_{2+}$ & $a_{2-}$ & $\phi_{c}$ & $\phi_{1+}$ & $\begin{array}{c}\phi_{1-}- \\
{[\mathrm{rad}]}\end{array}$ & $\phi_{2+}$ & $\phi_{2-}$ \\
\hline 12.213 & 12.243 & 12.183 & 12.274 & 12.150 & 2099 & 62 & 67 & 6.7 & 12.6 & 2.28 & -2.26 & 1.94 & -1.68 & 3.09 \\
\hline 7.054 & 7.084 & 7.026 & 7.113 & 6.990 & 1953 & 35 & 48 & 9.7 & 8.1 & -3.10 & -1.81 & -0.41 & -2.54 & 0.97 \\
\hline 6.943 & 6.972 & 6.912 & 7.007 & 6.988 & 1679 & 33 & 43 & 15 & 8.5 & -2.70 & -2.90 & 0.40 & 0.05 & 1.91 \\
\hline 8.141 & 8.169 & 8.110 & 8.194 & 8.081 & 1371 & 25 & 50 & 13 & 20 & -2.12 & -1.76 & -2.53 & 1.89 & 0.72 \\
\hline
\end{tabular}

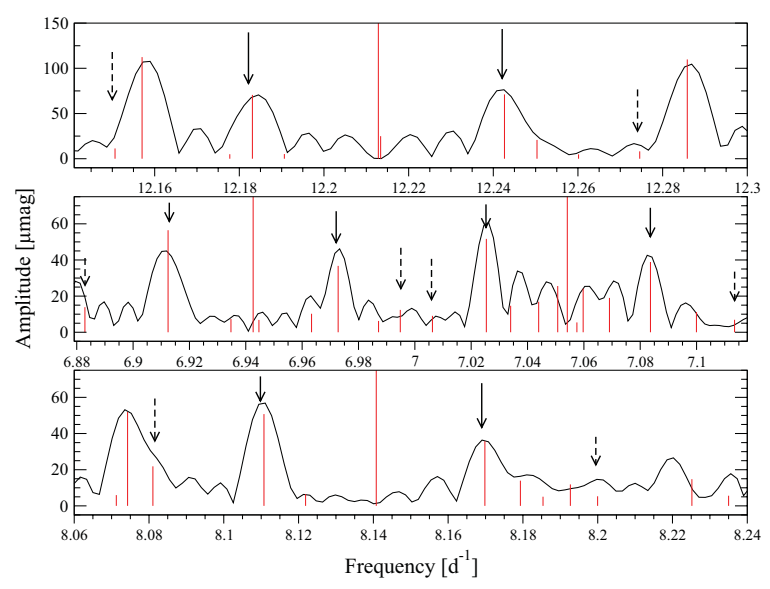

Figure 2. Identified candidate side-lobes at the four dominant frequencies. The solid line shows the Fourier transform after prewhitening with the four dominant frequencies and the vertical solid lines show the frequencies identified by SigSpec. The first-order side-lobes spaced by $0.03 \mathrm{~d}^{-1}$ are marked by solid-line arrows while the second-order side-lobes are marked by dashedline arrows.

noise of the residuals after prewhitening is $1.8 \mathrm{ppm}$, which transforms to a $\mathrm{S} / \mathrm{N}$ of 3.7 for the lowest-amplitude side-lobe. The amplitudes of the lower-than-central-frequency side-lobes $\left(a_{i-}\right)$ are systematically higher than those of the higher-than-central-frequency $\left(a_{i+}\right)$ side-lobes.

Candidate frequencies for the second-order side-lobes were found around the four dominant frequencies but no side-lobes were detected for the other frequencies. The amplitudes of these second-order side peak candidates range from only 6.7 to $21 \mathrm{ppm}$. We show that due to their low amplitudes and accordingly large relative errors, they are not sufficient to recover the RV curve purely from photometry.

Figure 2 shows the structure of side-lobes around the four dominant frequencies, with central frequencies subtracted. Table 1 lists the four dominant pulsation frequencies (column $\left.f_{c}\right)$, the identified side-lobes $\left(f_{i \pm}\right)$, the corresponding amplitudes $\left(\mathrm{a}_{i \pm}\right)$ and phases ( $\phi_{i \pm}$, zero point: beginning of CoRoT observations, JD 2454784.565).

\section{The orbit}

The binary nature of this star was discovered in the ESO LP185.D-0056 HARPS spectroscopy programme (led by E. Poretti). The light curve clearly shows a recurring brightening event with a period of 33.498 days. Thus, a spectroscopic campaign was organised with good phase coverage that confirmed this period as the binary orbit period. These spectra include 19 HERMES and five McDonald spectra. 


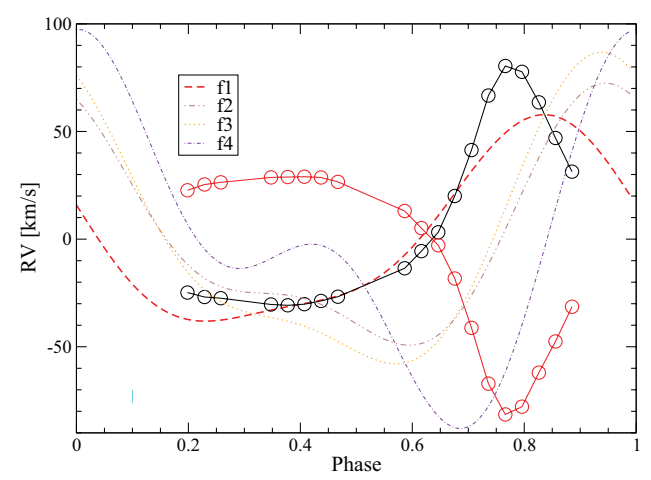

Figure 3. Comparison of the RV curves from spectroscopy to the RV curves from the frequency modulation. The spectroscopic RV measurements are corrected for $V_{0}$ and their errors are below $3 \mathrm{~km} \mathrm{~s}^{-1}$, which is of the order of the symbol size.

The radial velocities (RVs) were determined by fitting synthetic spectra to the observations using the software BinMag3 (by O. Kochukhov, www.astro.uu.ee/ oleg). First, the $v \sin i$ was determined by fitting synthetic spectra (both with $T_{\text {eff }}=6800 \mathrm{~K}$ and $\log g=$ $3.5)$ to the observations in which the lines of both components are well separated. In total, 60 measurements were averaged and the mean values of $v \sin i=41.4 \pm 1.5$ and $41.7 \pm 1.7 \mathrm{~km} \mathrm{~s}^{-1}$ respectively, were determined. Then, assuming $v \sin i$ for both components equal to $42 \mathrm{~km} \mathrm{~s}^{-1}$, we determined the RV curves for all spectra with the same software. For each spectrum, 16 frequency ranges were selected to fit the RVs. The standard deviations for the phases where the lines of the components are well separated are $1.5 \mathrm{~km} \mathrm{~s}^{-1}$ and for the phases where the lines are blended $\approx 3 \mathrm{~km} \mathrm{~s}^{-1}$. A preliminary fit to these RVs yielded an eccentricity of 0.48 and the projected semi-major axis of $65 R_{\odot}$.

The theoretical RV curves calculated from the frequencies (Table 1) are compared to the observed RVs in Fig. 3. For $f_{1}$ the agreement is acceptable, while for $f_{2}$ to $f_{4}$ the agreement is poor. Because the second order side-lobes have extremely low amplitudes, they are prone to significant relative errors. The frequency $f_{1}$ is consistent with the slightly lower mass star, which shows the LPV. We estimate that a noise level of less than $1 \mathrm{ppm}$ in the Fourier domain is required to reconstruct the RV curve purely from photometry for such low-amplitude $\delta$ Sct stars.

The brightening of the system occurs when the RVs of the components are at maximum separation. Thus, a tidal deformation and reflected light are likely to cause the brightening ("heartbeat effect").

\section{Acknowledgements}

M.H. and M.P. acknowledge financial support of the ESA PECS project 4000103541/ 11/NL/KML. M.H. is grateful to J. Benkö and L. Fosatti for valuable discussion. We are grateful to the observers, P. de Cat, P. Lampens, P.M. Arenal, J. Vos, and M. Rainer, for collecting the spectra.

\section{References}

Breger, M. 2000, ASP-CS, 210, 3

Pamyatnykh, A. A. 2000, ASP-CS, 210, 215

Reegen, P. 2007, A\&A, 467, 1353

Shibahashi, H. \& Kurtz, D. W. 2012, MNRAS, 422, 738

Thompson, S. E., Everett, M., Mullally, F., et al. 2012, ApJ, 753, 86 